\title{
Near and Far
}

The approach to clinical conundrums by an expert clinician is revealed through the presentation of an actual patient's case in an approach typical of a morning report. Similar to patient care, sequential pieces of information are provided to the clinician, who is unfamiliar with the case. The focus is on the thought processes of both the clinical team caring for the patient and the discussant.

This icon represents the patient's case. Each paragraph that follows represents the discussant's thoughts.

Adam Gray, MD², Sean Lockwood, MD², Aibek E. Mirrakhimov, MD¹, Allan C. Gelber, MD³, Reza Manesh, MD*

${ }^{1}$ Department of Medicine, University of Kentucky College of Medicine, Lexington, Kentucky; ${ }^{2}$ Department of Medicine, Lexington Veterans Affairs Medical Center, Lexington, Kentucky; ${ }^{3}$ Department of Medicine, Johns Hopkins Hospital and Johns Hopkins University School of Medicine, Baltimore, Maryland.

A previously healthy 30 -year-old woman presented to the emergency department with 2 weeks of weakness.

True muscle weakness must be distinguished from the more common causes of asthenia. Many systemic disorders produce fatigue, with resulting functional limitation that is often interpreted by patients as weakness. Initial history should focus on conditions producing fatigue, such as cardiopulmonary disease, anemia, connective tissue disease, depression or cachexia related to malignancy, infection, or other inflammatory states. Careful questioning may reveal evidence of dyspnea, poor exercise tolerance, or joint pain as an alternative to actual loss of muscle power. If true weakness is still suspected, attention should be focused on the pattern, onset, anatomic site, and progression of weakness. Muscle weakness is often characterized by difficulty with specific tasks, such as climbing stairs, rising from a chair, raising a hand, or using cutlery. The physical examination is critical in determining whether weakness is due to true loss of motor power. The differential diagnosis of weakness is broad and includes neurologic, infectious, endocrine, inflammatory, genetic, metabolic, and drug-induced etiologies.

She initially experienced 3 days of mild cramps and soreness in her thighs. She then developed weakness that began in her thighs and progressed to involve her lower legs and upper and lower arms. She had difficulty combing her hair. She required the use of her arms to get up from a chair. She grasped onto objects to aid in ambulation around the house. In addition, she described 1 year of moderate fatigue

\footnotetext{
*Address for correspondence: Reza Manesh, MD, Division of General Internal Medicine, Johns Hopkins Hospital, 600 N. Wolfe Street / Meyer 8-34D, Baltimore, MD 21287; Telephone: 412-708-6944; Fax: 410-502-0923; E-mail: rsedigh1@jhmi.edu

Published online first December 20, 2017.
}

Received: July 4, 2017; Revised: August 27, 2017;

Accepted: September 1, 2017

(c) 2018 Society of Hospital Medicine DOI 10.12788/jhm.2893 but no fever, weight loss, dyspnea, dysphagia, visual changes, paresthesias, bowel or bladder incontinence, back pain, or preceding gastrointestinal or respiratory illness. She had experienced diffuse intermittent hives, most prominent in her chest and upper arms, for the past several weeks.

History certainly supports true weakness but will need to be confirmed on examination. The distribution began as proximal but now appears diffuse. The presence of myalgia and cramping raises the possibility of noninflammatory myopathies, which are usually more insidious in onset. A severe electrolyte disturbance would be possible, based on the diffuse nature of weakness that was preceded by cramping. The distribution of weakness and lack of bowel or bladder incontinence is reassuring and suggests against a spinal cord disorder; however, a high index of suspicion must be maintained for myelopathy because delayed treatment might result in irreversible paralysis.

The patient's course also includes hives. Common causes of hives include infections and allergic reactions to medications, foods, and insect stings. Urticaria may also result from systemic disorders, such as vasculitis, lupus, lymphoma, mastocytosis, and paraproteinemias, which can be associated with weakness and fatigue. Although severe weakness in combination with hives makes an infectious and allergic reaction less likely, we still seek to ascertain if the evolving chief complaints of weakness and hives are the result of a single unifying and evolving multisystem disorder or are distinct and unrelated processes.

Her past medical history included fibromyalgia, kidney stones, and gastroesophageal reflux disease. One week prior to presentation, she was prescribed prednisone $60 \mathrm{mg}$ daily for the treatment of hives; the dose had been tapered to $40 \mathrm{mg}$ at presentation, with mild improvement of hives. She recently started doxepin for fibromyalgia and insomnia. She lived at home with her husband and 8-year-old child. She worked as a clerk in a pest control office and denied any pesticide exposure. She denied tobacco, alcohol, or illicit drug use. Her family history included systemic lupus erythematosus (SLE) in her mother and maternal aunt. 
Glucocorticoids are associated with myopathy; however, the weakness preceded steroid therapy. Thus, unless there was unknown exposure to high-dose steroid medication to treat recurrent episodes of urticaria earlier in her course, glucocorticoid-related myopathy is unlikely. Fibromyalgia might cause the perception of weakness from pain. However, the history of difficulty combing her hair and rising from a chair suggests actual loss of motor power. The side effects of her medications, such as newly started doxepin, must be reviewed. A family history of SLE raises concern for rheumatologic conditions; however, one might expect improvement with steroid therapy.

On physical examination, her temperature was $36.9^{\circ} \mathrm{C}$, blood pressure $126 / 93 \mathrm{mmHg}$, pulse 81 beats per minute, respiratory rate 16 breaths per minute, and oxygen saturation $100 \%$ on ambient air. Her cardiopulmonary examination was normal. Her abdomen was nontender and without hepatosplenomegaly. Her strength was 2 out of 5 in proximal and distal legs, bilaterally, and 4 out of 5 in proximal and distal upper extremities. She had normal muscle tone without fasciculations or atrophy. Her joints were without edema, erythema, or impaired range of motion. She had normal sensation to light touch in arms and legs. Her reflexes were 2+ in the patellar, Achilles, and brachioradialis tendons. She had no lymphadenopathy, mucosal ulcerations, or alopecia. A skin examination revealed smooth, slightly elevated, and faded pink wheals that were diffuse but most prominent in upper arms and chest.

Physical examination confirms the presence of true muscle weakness. The differential diagnosis is narrowed by several findings, both positive and negative, elicited in the examination. The diffuse nature of the weakness eliminates focal central nervous system lesions, such as stroke, intracranial mass lesions, or demyelinating white matter foci. Combining this finding with normal reflexes and history of preceding myalgias makes electrolyte-induced and inflammatory (eg, polymyositis) myopathies more likely. The normal deep tendon reflexes and the absence of a delayed relaxation phase lower the likelihood of hypothyroidism.

Diseases originating from the neuromuscular junction, such as myasthenia gravis, may also present with weakness and normal reflexes, although this pattern of weakness would be atypical; myasthenia gravis classically presents with fatigable weakness and ocular findings of diplopia and/or ptosis. First-tier testing should include a complete blood count to evaluate for eosinophilia, comprehensive metabolic panel, and urinalysis for myoglobinuria, thyroid stimulating hormone, and muscle enzymes.

Results of a complete blood count demonstrated a leukocyte count of $16.1 \mathrm{k} / \mathrm{uL}$ with $82 \%$ neutrophils, $13 \%$ lymphocytes, $5 \%$ monocytes, and $0 \%$ eosinophils. Hemoglobin was $13.2 \mathrm{~g} / \mathrm{dL}$, and platelet count $226 \mathrm{k} / \mathrm{uL}$. Sodium was $136 \mathrm{mmol} / \mathrm{L}$, potassium $1.5 \mathrm{mmol} / \mathrm{L}$, chloride $115 \mathrm{mmol} / \mathrm{L}$, bicarbonate $12 \mathrm{mmol} / \mathrm{L}$, blood urea nitrogen $26 \mathrm{mg} / \mathrm{dL}$, creatinine $1.0 \mathrm{mg} / \mathrm{dL}$ (baseline creatinine: 0.6 ), and glucose 102 $\mathrm{mg} / \mathrm{dL}$. Calcium was $9.4 \mathrm{mg} / \mathrm{dL}$, magnesium $2.6 \mathrm{mg} / \mathrm{dL}$, phosphorus $1.8 \mathrm{mg} / \mathrm{dL}, \mathrm{CK} 501 \mathrm{U} / \mathrm{L}$ (normal: 40-230), and TSH $5.48 \mathrm{ulU} / \mathrm{mL}$ (normal: 0.5-4). Aspartate aminotransferase was $64 \mathrm{U} / \mathrm{L}$, alanine aminotransferase $23 \mathrm{U} / \mathrm{L}$, alkaline phosphatase $66 \mathrm{U} / \mathrm{L}$, bilirubin $0.9 \mathrm{mg} / \mathrm{dL}$, albumin $3.8 \mathrm{~g} / \mathrm{dL}$, and total protein $8.7 \mathrm{~g} / \mathrm{dL}$ (normal: 6.2-7.8). Human immunodeficiency virus antibody screen was negative. An electrocardiogram revealed normal sinus rhythm, flattened T waves, and prominent $U$ waves.

Potassium losses are classically categorized into 1 of 3 groups: renal losses, gastrointestinal losses, or transcellular shifts. Without a clear history of diuretic use, renal losses may not be apparent on history and examination. In contrast, gastrointestinal losses are almost always evidenced by a history of vomiting and/or diarrhea, with rare exceptions, including unreported laxative abuse or surreptitious vomiting. Transcellular potassium shifts can be seen in states of increased insulin or beta-adrenergic activity and alkalosis and result from both primary and secondary causes of hypokalemic periodic paralysis.

The presence of a reduced serum bicarbonate and elevated chloride concentration suggests a normal anion gap metabolic acidosis. Many conditions associated with normal anion gap metabolic acidosis are evident by history, such as diarrhea. In enigmatic cases such as this, it will be important to take a stepwise approach that includes an evaluation for urinary potassium losses and assessment of acid-base status. An unexplained normal anion gap metabolic acidosis combined with hypokalemia raises suspicion for a distal renal tubular acidosis (RTA). Additional testing to evaluate for a possible RTA should include the assessment of urinary electrolytes and urinary $\mathrm{pH}$. The hypokalemia explains her weakness, but the etiology of such profound hypokalemia is not evident, nor is it clear how it relates to her hives.

The severity of the hypokalemia, combined with electrocardiogram changes, necessitates rapid intravenous potassium repletion, telemetry monitoring, and frequent serum potassium measurement. Treatment of her metabolic acidosis is more nuanced and depends upon both the severity of disturbance and the suspicion of whether the etiology is transcellular shift, potassium depletion, or both.

Urine studies demonstrated a urine specific gravity of 1.006 (normal: 1.001-1.030), urine $\mathrm{pH}$ was 6.5 (normal: 5-6.5), trace leukocyte esterase, negative nitrite, $30 \mathrm{mg} / \mathrm{dL}$ of protein (normal: <15), sodium $64 \mathrm{mmol} / \mathrm{L}$ (normal: 40-220), potassium $17 \mathrm{mmol} / \mathrm{L}$ (normal: 25-125), and chloride 71 $\mathrm{mmol} / \mathrm{L}$ (normal: 110-250). Urine microscopy demonstrated 3 red blood cells per high power field (normal: 0-1), 4 white blood cells per high power field (normal: 0-4), 4+ bacteria per high power field, and no red blood cell casts. Urine protein-to-creatinine ratio was 1.6. C3 and C4 complement levels were $53 \mathrm{mg} / \mathrm{dL}$ (normal: 80-165) and $12 \mathrm{mg} / \mathrm{dL}$ (normal: 1549), respectively. C-reactive protein was $<0.5$ (normal: $0-0.9$ ), and erythrocyte sedimentation rate was $16 \mathrm{~mm} /$ hour (normal: 0-20). 
A calculation of the urine anion gap (UAG; [urine sodium + urine potassium] - urine chloride) yields a UAG of $10 \mathrm{mq} / \mathrm{L}$. A positive UAG, together with a nongap metabolic acidosis, should prompt the consideration of RTA. The normal renal response to acidosis is to reduce the urine $\mathrm{pH}$ to less than 5.3 through an increase in hydrogen ion excretion in the form of ammonium. A urine $\mathrm{pH}$ of 6.5 is highly suggestive of type 1 (distal) RTA and its associated impairment of distal acidification. Treatment with sodium bicarbonate to correct the acidosis and associated complications is warranted.

A distal RTA would account for her past medical history of renal stones. Acidemia promotes both increased calcium phosphate release from bone (with subsequent hypercalciuria) and enhanced citrate reabsorption in the proximal renal tubules, leading to decreased urinary citrate. Citrate inhibits calcium stone formation. The increased calcium load to renal tubules in addition to decreased urinary citrate both lead to increased precipitation of calcium stones in the genitourinary tract.

A diagnosis of distal RTA should prompt evaluation for specific etiologies, such as Sjögren's syndrome or SLE. While not diagnostic of any specific condition, low C3 and C4 levels suggest immune complex formation with related complement consumption, contributing to hypocomplementemia. The diagnosis of RTA may occur among patients with Sjögren's syndrome in the absence of overt evidence of sicca syndrome (xerostomia and keratoconjunctivitis sicca). Other etiologies of distal RTA include conditions leading to hypercalciuria, such as hyperparathyroidism and idiopathic hypercalciuria, hereditary causes, toxins such as toluene, and drugs such as amphotericin $\mathrm{B}$, lithium carbonate, and ibuprofen.

Her antinuclear antibody titer was $>1: 1280$ (normal: $<80$ ). Anti-SSA and -SSB antibodies were both positive, with a titer $>100$ (normal: $<20$ ). Rheumatoid factor was positive at $22 \mathrm{IU} / \mathrm{mL}$ (normal: 0-14). Anti-smith, anti-double stranded DNA, and anti-ribonucleoprotein antibodies were negative.

Sjögren's syndrome appears to be the ultimate etiology of this patient's distal RTA. The diagnosis of Sjögren's is more classically made in the presence of lacrimal and/or salivary dysfunction and confirmed with compatible autoantibodies. In the absence of dry eyes or dry mouth, attention should be focused on her skin findings. Cutaneous vasculitis does occur in a small percentage of Sjögren's syndrome cases. Urticarial lesions have been reported in this subset, and skin biopsy would further support the diagnosis.

Treatment of Sjögren's syndrome with immunosuppressive therapy may ameliorate renal parenchymal pathology and improve her profound metabolic disturbances.

On further questioning, she described several months of mild xerostomia, which resulted in increased consumption of fluids. She did not have keratoconjunctivitis sicca. Biopsy of her urticarial rash demonstrated a leukocytoclastic vasculitis with eosinophilic infiltration (Figure 1). Renal biopsy with hematoxylin and eosin staining, immunofluorescence, and electron microscopy demonstrated an immune complex-mediated glomerulonephritis and moderate tubulointerstitial nephritis (Figure 2). A diagnosis of Sjögren's syndrome was made based on the patient's xerostomia, high titers of antinuclear antibodies, SSA and SSB antibodies, positive rheumatoid factor, hypocomplementemia, and systemic manifestations associated with Sjögren's syndrome, including distal RTA, nephrolithiasis, and hives, with histologic evidence of leukocytoclastic vasculitis.

She received aggressive potassium and bicarbonate repletion and, several days later, had normalization of both. Her weakness and myalgia rapidly improved concomitantly with the correction of her hypokalemia. Five days later she was ambulating independently and discharged with potassium

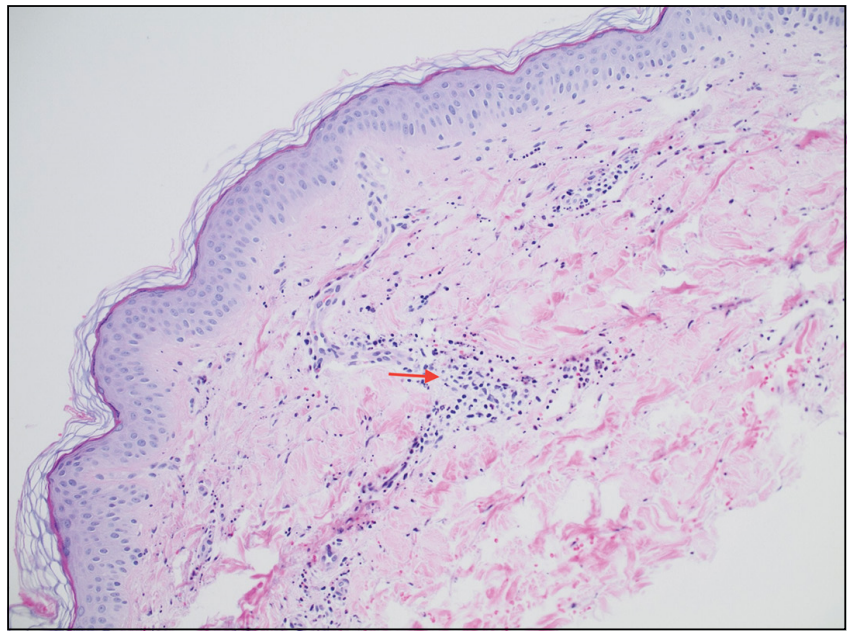

FIG 1. Biopsy specimen of the skin (hematoxylin and eosin, x20). Epidermis and dermis with mixed vascular inflammatory infiltrate, consistent with leukocytoclastic vasculitis.

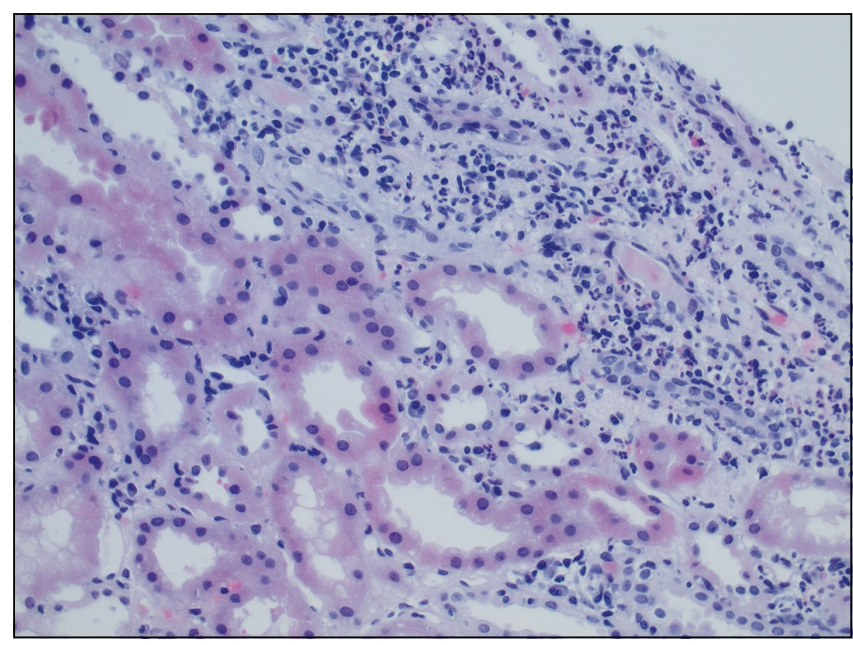

FIG 2. Biopsy specimen of the kidney (hematoxylin and eosin, $x 40$ ). A mixed interstitial inflammatory infiltrate with lymphocytes, polymorphonuclear neutrophils, and eosinophils is demonstrated, which is consistent with tubulointerstitial nephritis. 
citrate and prednisone therapy. She had improved fatigue and rash at a 1-month follow-up with rheumatology. As an outpatient, she was started on azathioprine and slowly tapered off her steroids. Over the next several months, she had normal potassium, bicarbonate, and renal function, although she did require lithotripsy for an obstructive renal stone.

\section{COMMENTARY}

RTA should be considered in the differential diagnosis of an unexplained normal anion gap metabolic acidosis. There are 3 major types of RTAs, with different characteristics. In type 1 (distal) RTA, the primary defect is impaired distal acidification of the urine. Distal RTA commonly presents with hypokalemia, calciuria (often presenting as renal stones), and a positive UAG. ${ }^{1}$ In type 2 (proximal) RTA, the primary defect is impaired bicarbonate reabsorption, leading to bicarbonate wasting in the urine. Proximal RTAs can be secondary to an isolated defect in bicarbonate reabsorption or generalized proximal renal tubule dysfunction (Fanconi syndrome). ${ }^{1}$ A type 4 RTA is characterized by hypoaldosteronism, presenting usually with a mild nonanion gap metabolic acidosis and hyperkalemia. This patient's history of renal stones, hypokalemia, and positive UAG supported a type 1 (distal) RTA. Distal RTA is often idiopathic, but initial evaluation should include a review of medications and investigation into an underlying systemic disorder (eg, plasma cell dyscrasia or autoimmune disease). This would include eliciting a possible history of xerostomia and xerophthalmia, together with testing of SSA (Ro) and SSB (La) antibodies, to assess for Sjögren's syndrome. In addition, checking serum calcium to assess for hyperparathyroidism or familial idiopathic hypercalciuria and a review of medications, such as lithium and amphotericin, ${ }^{1}$ may uncover other secondary causes of distal RTA.

While Sjögren's syndrome primarily affects salivary and lacrimal glands, leading to dry mouth and dry eyes, respectively, extraglandular manifestations are common, with fatigue and arthralgia occurring in half of patients. Extra-glandular involvement also often includes the skin and kidneys but can affect several other organ systems, including the central nervous system, heart, lungs, bone marrow, and lymph nodes. ${ }^{2}$

There are many cutaneous manifestations of Sjögren's syndrome. ${ }^{3}$ Xerosis, or xeroderma, is the most common and is characterized by dry, scaly skin. Cutaneous vasculitis can occur in 10\% of patients with Sjögren's syndrome and often presents with palpable purpura or diffuse urticarial lesions, as in our patient. ${ }^{4}$ Erythematous maculopapules and cryoglobulinemic vasculitis may also occur. ${ }^{4} \mathrm{~A}$ less common skin manifestation is annular erythema, presenting as an indurated, ring-like lesion. ${ }^{5}$

Chronic tubulointerstitial nephritis is the most common renal manifestation of Sjögren's syndrome. ${ }^{6}$ This often pre-sents with a mild elevated serum creatinine and a distal RTA, leading to hypokalemia, as in the case discussed. Distal RTA is well described, occurring in one-quarter of patients with Sjögren's syndrome. The pathophysiology leading to distal RTA in Sjögren's syndrome is thought to arise from autoimmune in- jury to the $\mathrm{H}(+)$-ATPase pump in the renal collecting tubules, leading to decreased distal proton secretion. 8,9 Younger adults with Sjögren's syndrome, in the third and fourth decades of life, have a predilection to develop tubulointerstitial inflammation, distal RTA, and nephrolithiasis, as in the present case. ${ }^{6}$ Sjögren's syndrome less commonly presents with membranoproliferative glomerulonephritis or membranous nephropathy. ${ }^{10,11}$ Cryoglobulinemia-associated hypocomplementemia and glomerulonephritis may also occur with Sjögren's syndrome, yet glomerular lesions are less common than is tubulointerstitial inflammation. The patient discussed had proteinuria and evidence of immune complex-mediated glomerulonephritis.

Treatment of sicca symptoms is generally supportive. It includes artificial tears, encouragement of good hydration, salivary stimulants, and maintaining good oral hygiene. Pilocarpine, a cholinergic parasympathomimetic agent, is approved by the Food and Drug Administration to treat dry mouth associated with Sjögren's syndrome. The treatment of extraglandular manifestations depends on the organ(s) involved. More severe presentations, such as vasculitis and glomerulonephritis, often require immunosuppressive therapy with systemic glucocorticoids, cyclophosphamide, azathioprine, or other immunosuppressive agents, ${ }^{12}$ including rituximab. RTA often necessitates treatment with oral bicarbonate and supplemental potassium repletion.

The base rate of disease (ie, prevalence of disease) influences a diagnostician's pretest probability of a given diagnosis. The discussant briefly considered rare causes of hives (eg, vasculitis) but appropriately fine-tuned their differential for the patient's hypokalemia and RTA. Once the diagnosis of Sjögren's syndrome was made with certainty, the clinician was able to revisit the patient's rash with a new lens. Urticarial vasculitis suddenly became a plausible consideration, despite its rarity (compared to allergic causes of hives) because of the direct link to the underlying autoimmune condition, which affected both the proximal muscles and distal nephrons.

\section{TEACHING POINTS}

- Evaluation of patients with weakness starts with determining true muscle weakness (ie, pathology involving the brain, spinal cord, peripheral nerve, neuromuscular junction, and/or muscle) from asthenia.

- Distal RTA should be considered in patients with a nonanion gap metabolic acidosis and hypokalemia.

- Sjögren's syndrome has many extraglandular clinical manifestations, including vasculitis, urticaria, tubulointerstitial renal inflammation, glomerulonephritis, and lymphoma.

\section{Acknowledgment}

The authors thank Virgilius Cornea, MD, for his interpretation of the pathologic images

Disclosure: Dr. Manesh is supported by the Jeremiah A. Barondess Fellowship in the Clinical Transaction of the New York Academy of Medicine, in collaboration with the Accreditation Council for Graduate Medical Education (ACGME). The authors declare no conflicts of interests. 


\section{References}

1. Rodríguez Soriano J. Renal tubular acidosis: the clinical entity. J Am Soc Nephrol. 2002;13(8):2160-2170.

2. Asmussen K, Andersen V, Bendixen G, Schiødt M, Oxholm P. A new model for classification of disease manifestations in primary Sjögren's syndrome: evaluation in a retrospective long-term study. J Intern Med. 1996;239(6): 475-482.

3. Kittridge A, Routhouska SB, Korman NJ. Dermatologic manifestations of Sjögren syndrome. J Cutan Med Surg. 2011;15(1):8-14.

4. Ramos-Casals M, Anaya JM, García-Carrasco M, et al. Cutaneous vasculitis in primary Sjögren syndrome: classification and clinical significance of 52 patients. Medicine (Baltimore). 2004; 83(2):96-106.

5. Katayama I, Kotobuki Y, Kiyohara E, Murota H. Annular erythema associated with Sjögren's syndrome: review of the literature on the management and clinical analysis of skin lesions. Mod Rheumatol. 2010;20(2):123-129.

6. Maripuri S, Grande JP, Osborn TG, et al. Renal involvement in primary Sjögren's syndrome: a clinicopathologic study. Clin J Am Soc Nephrol. 2009;4(9):1423-1431.
7. Pun KK, Wong CK, Tsui EY, Tam SC, Kung AW, Wang CC. Hypokalemic periodic paralysis due to the Sjögren syndrome in Chinese patients. Ann Intern Med. 1989;110(5):405-406.

8. Cohen EP, Bastani B, Cohen MR, Kolner S, Hemken P, Gluck SL. Absence of $\mathrm{H}(+)$-ATPase in cortical collecting tubules of a patient with Sjogren's syndrome and distal renal tubular acidosis. J Am Soc Nephrol. 1992;3(2):264-271.

9. Bastani B, Haragsim L, Gluck S, Siamopoulos KC. Lack of H-ATPase in distal nephron causing hypokalaemic distal RTA in a patient with Sjögren's syndrome. Nephrol Dial Transplant. 1995;10(6):908-909.

10. Cortez MS, Sturgill BC, Bolton WK. Membranoproliferative glomerulonephritis with primary Sjögren's syndrome. Am J Kidney Dis. 1995;25(4): 632-636

11. Baba A, Hara S, Sato Y, Yamada K, Fujimoto S, Eto T. [Three patients with nephrotic syndrome due to membranous nephropathy complicated by Sjögren's syndrome]. Nihon Jinzo Gakkai Shi. 2005;47(8):882-886.

12. Thanou-Stavraki A, James JA. Primary Sjogren's syndrome: current and prospective therapies. Semin Arthritis Rheum. 2008;37(5):273-292. 